\title{
Anti-phase synchronization and symmetry-breaking bifurcation of impulsively coupled oscillators
}

\author{
Haibo Jianga*, Yang Liu ${ }^{\mathrm{b}}$, Liping Zhang ${ }^{\mathrm{a}}$, Jianjiang $\mathrm{Yu}^{\mathrm{c}}$ \\ a School of Mathematics and Statistics, Yancheng Teachers University, Yancheng 224002, China \\ ${ }^{\mathrm{b} S c h o o l ~ o f ~ E n g i n e e r i n g, ~ R o b e r t ~ G o r d o n ~ U n i v e r s i t y, ~ G a r t h d e e ~ R o a d, ~ A b e r d e e n ~ A B 10 ~ 7 G J, ~ U K ~}$ \\ ${ }^{\mathrm{c} S}$ School of Information Science and Technology, Yancheng Teachers University, Yancheng 224002, China
}

\begin{abstract}
This paper studies the synchronization in two mechanical oscillators coupled by impacts which can be considered as a class of state-dependent impulsively coupled oscillators. The two identical oscillators are harmonically excited in a counter phase, and the synchronous (antiphase synchronization) and the asynchronous motions are considered. One- and two-parameter bifurcations of the system have been studied by varying the amplitude and the frequency of external excitation. Numerical simulations show that the system could exhibit complex phenomena, including symmetry and asymmetry periodic solutions, quasi-periodic solutions and chaotic solutions. In particular, the regimes in anti-phase synchronization are identified, and it is found that the symmetry-breaking bifurcation plays an important role in the transition from synchronous to asynchronous motion.
\end{abstract}

Keywords: impact oscillators, state-dependent impulsively coupled oscillators, anti-phase synchronization, symmetry-breaking bifurcation

\section{Introduction}

Synchronization phenomena are ubiquitous in nature, and they have been extensively studied by using different methods (e.g. [1-3]). Nowadays, various types of synchronization have been

${ }^{*}$ Corresponding author. Tel.:+86 515 88239915, E-mail address: yctcjhb@126.com (H. Jiang), y.liu8@rgu.ac.uk (Y. Liu), yctczlp@126.com (L. Zhang), yujianjiang@126.com (J. Jiang). 
investigated, including complete synchronization [4], phase synchronization [5], lag synchronization $[6,7]$, generalized synchronization [8], and so on. In particular, a special phenomenon in the coupled oscillators called anti-synchronization has attracted significant attention from researchers, e.g. $[9,10]$. When the coupled oscillators achieve anti-synchronization, their states have the same absolute values but opposite signs. The scientist Huygens first observed the anti-synchronization in two pendulum clocks [11], and this phenomenon was also known as the anti-phase synchronization (APS), e.g. $[12,13]$.

Since the transition of synchronization can be revealed through dynamical analysis, the synchronization of coupled oscillators was always investigated by studying its dynamics, e.g. [14-16]. In [14], Rene investigated different dynamical states of synchronization for a ring of four mutually inertia coupled self-sustained electrical systems which were described by the coupled Rayleigh-Duffing equations. He also studied the stability properties of periodic solutions and the transition boundaries between different dynamical states by using the Floquet theory. The dynamical behaviors and synchronization of a ring of mutually coupled Van der Pol oscillators were studied by Barrón and Sen in [15]. Later on, Perlikowski et al. [16] investigated the dynamics of a ring of unidirectionally coupled autonomous Duffing oscillators which indicated that although the individual uncoupled oscillator has one globally stable equilibrium, the response of the coupled oscillators could evolve into periodic, quasi-periodic, and chaotic motions when the coupling strength increases. In [17], Kapitaniak et al. considered the dynamics of two planar elastic pendula mounted on a horizontally excited platform in order to identify any possible synchronous states.

The couplings between the oscillators reviewed above are continuous, while not all the coupled systems are continuous, e.g. the species-food model in biology, the information transfer and exchange in ants, and the model of integrated circuit. The impulsively coupled oscillators which interact with each other at discrete time is a counter example of continuous coupling. They have drawn considerable attention from researchers because of their board applications in image processing, circuit design and implement, and the others (e.g. [18-21]). Han et al. [18] studied a class of impulsively coupled complex dynamical systems and established several criteria regarding to the eigenvalues and the eigenvectors of the coupling matrix for synchronization of such systems. Yang et al. studied a class of impulsively coupled complex switched networks and their robust synchronization in terms of parametric uncertainties and time-varying delays in [19]. Jiang and Bi introduced the concept of partial contraction theory of impulsive systems 
and investigated the synchronization problem of impulsively coupled oscillators in [20]. Jiang et al. [21] studied the complex dynamics of a non-smooth system which was unidirectionally impulsively coupled by three Duffing oscillators in a ring structure.

Recently, synchronization of various coupled dynamical systems have been extensively considered. For example, in [22-24], the impulsive control protocols have been introduced for networked linear and nonlinear dynamical systems. Blazejczyk-Okolewska et al. [25] studied the dynamics of two mechanical oscillators coupled by impacts and observed the anti-phase synchronization phenomenon when the distance between these oscillators exceeded some critical thresholds. In [26-29], the dynamics of multi-degree-of-freedom vibro-impact systems has been studied by using numerical simulations and qualitative analysis. This paper will use the physical model in [25] to study the dynamics and the synchronization of impulsively coupled oscillators under variations of amplitude and frequency of external excitation. In particular, the transition between the synchronous (APS) and asynchronous motions will be analyzed. One- and twoparameter bifurcation analysis will be conducted for investigating the relationship between the dynamic response and the synchronization phenomenon of the system. The symmetry-breaking bifurcation will be discussed in order to emphasize its role on the transition from synchronous to asynchronous response.

The remainder of this paper is organized as follows. In Section 2, the physical model of the impulsively coupled oscillators is presented. Event-based hybrid system modeling and stability analysis are given in Section 3. In Section 4, one- and two-parameter bifurcations and synchronization analysis of the system are analyzed, and then conclusions are given in Section 5.

\section{Physical model}

The physical model of the impulsively coupled oscillators is shown in Fig. $1 . X_{1}$ and $X_{2}$ are displacements of mass 1 and 2, respectively. Both masses are connected to the supporting base by two identical springs with stiffness $k$ and two identical linear viscous dampers with damping coefficient $c$. The external forcing on the masses is harmonic with amplitude $F$ and frequency $\Omega$ in a counter phase (i.e. $\varphi=\pi$, where $\varphi$ is the phase shift of external forcing). The initial gaps between the masses and the origin point $X=0$ are $E$. The impacts of these two masses are assumed to be rigid and described by a restitution coefficient $r$. It should be noted that 
the coupling between these two oscillators is non-continuous so that they can be described as state-dependent impulsive coupling.

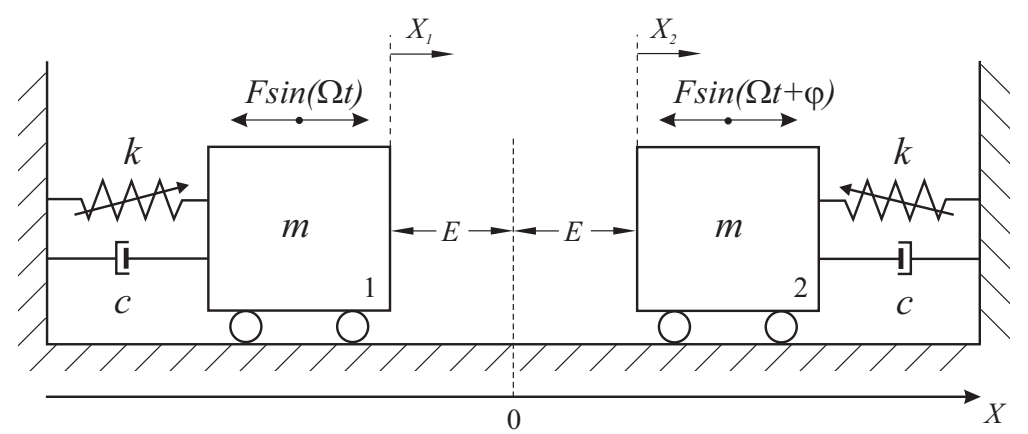

Fig. 1. Physical model of the state-dependent impulsively coupled oscillators (adapted from [25]).

Without impacts, the equations of motion for the impulsively coupled oscillators can be written as

$$
\left\{\begin{array}{l}
m \ddot{X}_{1}+c \dot{X}_{1}+k X_{1}^{3}=F \sin (\Omega t), \\
m \ddot{X}_{2}+c \dot{X}_{2}+k X_{2}^{3}=F \sin (\Omega t+\pi),
\end{array} \text { when } X_{2}-X_{1}>0 .\right.
$$

Using the rule of restitution coefficient and the law of conservation of momentum, the impact equations of the system are governed by

$$
\left\{\begin{array}{l}
\dot{X}_{2}^{+}-\dot{X}_{1}^{+}=-r\left(\dot{X}_{2}^{-}-\dot{X}_{1}^{-}\right), \\
m\left(\dot{X}_{1}^{+}-\dot{X}_{1}^{-}\right)+m\left(\dot{X}_{2}^{+}-\dot{X}_{2}^{-}\right)=0,
\end{array} \text { when } X_{2}-X_{1}=0\right.
$$

where $\dot{X}_{i}(i=1,2)$ is the differentiation of $X_{i}$ with respect to the time $t$, and $\dot{X}_{i}^{-}$and $\dot{X}_{i}^{+}$ represent the velocities of the $i$ th oscillator before and after impact, respectively.

We introduce the following non-dimensional variables and parameters: $\tau=\Omega_{n} t, \Omega_{n}=$ $\sqrt{k / m} y_{0}, x_{1}=X_{1} / y_{0}, x_{2}=X_{2} / y_{0}, e=E / y_{0}, \xi=c /\left(2 m \Omega_{n}\right), a=F /\left(k y_{0}^{3}\right), \omega=\Omega / \Omega_{n}$, where $y_{0}$ is some arbitrary reference distance. Then the non-dimensional equations of motion are rewritten as

$$
\begin{aligned}
& \left\{\begin{array}{l}
x_{1}^{\prime \prime}+2 \xi x_{1}^{\prime}+x_{1}^{3}=a \sin (\omega \tau), \\
x_{2}^{\prime \prime}+2 \xi x_{2}^{\prime}+x_{2}^{3}=a \sin (\omega \tau+\pi),
\end{array} \text { when } x_{2}-x_{1}>0,\right. \\
& \left\{\begin{array}{l}
\left(x_{1}^{\prime}\right)^{+}=\left((1-r)\left(x_{1}^{\prime}\right)^{-}+(1+r)\left(x_{2}^{\prime}\right)^{-}\right) / 2, \\
\left(x_{2}^{\prime}\right)^{+}=\left((1+r)\left(x_{1}^{\prime}\right)^{-}+(1-r)\left(x_{2}^{\prime}\right)^{-}\right) / 2,
\end{array} \text { when } x_{2}-x_{1}=0 .\right.
\end{aligned}
$$


It should be noted that $v_{1}$ and $v_{2}$ are defined as the differentiations of $x_{1}$ and $x_{2}$ with respect to the non-dimensional time $\tau$, respectively, which will be used in the following numerical simulations. In addition, the following definition is given.

Definition 1. The impulsively coupled oscillators are in APS if $\lim _{\tau \rightarrow+\infty}\left|x_{1}+x_{2}\right|=0$ and $\lim _{\tau \rightarrow+\infty}\left|v_{1}+v_{2}\right|=0$.

\section{Event-based hybrid system modeling and stability analysis}

In this section, we transform the system (3) into vector fields, event functions and jump functions by using the modeling approach of the event-based hybrid system studied in [30,31], and provide the method for determining the stability of periodic solutions.

Let

$$
y=\left(\begin{array}{l}
y_{1} \\
y_{2} \\
y_{3} \\
y_{4} \\
y_{5}
\end{array}\right)=\left(\begin{array}{c}
x_{1} \\
x_{1}^{\prime} \\
x_{2} \\
x_{2}^{\prime} \\
(\omega \tau) \bmod (2 \pi)
\end{array}\right)
$$

represent the state of the state-dependent impulsively coupled oscillators in (3). Then the smooth motion of the system is governed by the vector field

$$
f_{\text {smooth }}(y)=\left(\begin{array}{c}
y_{2} \\
-2 \xi y_{2}-y_{1}^{3}+a \sin \left(y_{5}\right) \\
y_{4} \\
-2 \xi y_{4}-y_{3}^{3}+a \sin \left(y_{5}+\pi\right) \\
\omega
\end{array}\right) .
$$

Impact occurs when

$$
h_{\text {impact }}(y)=y_{3}-y_{1}=0
$$

resulting in a discontinuous jump in state given by the state jump function

$$
g_{\text {impact }}(y)=\left(\begin{array}{c}
y_{1} \\
\left((1-r) y_{2}+(1+r) y_{4}\right) / 2 \\
y_{3} \\
\left((1+r) y_{2}+(1-r) y_{4}\right) / 2 \\
y_{5}
\end{array}\right) .
$$


Furthermore, a discontinuous jump in the phase coordinate $y_{5}$ occurs when

$$
h_{\text {phase }}(y)=2 \pi-y_{5}=0
$$

which corresponds to the state jump function

$$
g_{\text {phase }}(y)=\left(\begin{array}{c}
y_{1} \\
y_{2} \\
y_{3} \\
y_{4} \\
y_{5}-2 \pi
\end{array}\right) \text {. }
$$

A periodic trajectory of hybrid dynamical systems (e.g. impulsive switched systems) can be characterized by a sequence of triplets in the form $(f, h, g)$ which includes a solution segment governed by the vector field $f$ terminating on the event surface $h$ and connecting to the next solution segment by using the state jump function $g$. It is therefore that the periodic trajectories of the system in (3) consist of two signatures, $I_{1}=\left(f_{\text {smooth }}, h_{\text {impact }}, g_{\text {impact }}\right)$ and $I_{2}=\left(f_{\text {smooth }}, h_{\text {phase }}, g_{\text {phase }}\right)$. For a given signature of the periodic trajectory, the Jacobian matrix of the smooth part uses the monodromy matrix of the smooth trajectory, and the Jacobian matrices of the impact and the phase use their corresponding saltation matrices [32]. Using such an approach, one can get the periodic solution and the corresponding Jacobian matrix of the periodic trajectory. Four basic types of bifurcations including saddle-node (SN), symmetry-breaking (SB)/pitchfork, period-doubling (PD), and Neimark-Sacker (NS) bifurcations are responsible for destabilization of the observed periodic solutions. The bifurcations are analyzed by using the continuation toolbox CoCo $[33,34]$, which is a general-purpose tool for continuation and bifurcation analysis of smooth and non-smooth dynamical systems. For the system in (3), CoCo can distinguish the types of bifurcations based on the Floquet theory (see [34] for details) by calculating four Floquet multipliers of periodic solutions. When all multipliers are within the unit circle, the considered periodic solution is stable. As the two impact oscillators are identical and symmetric, SN or SB bifurcation occurs when one of the real multipliers leaves the unit circle through +1 , and -1 for PD bifurcation, or two pairs of complex multipliers leave the circle in the case of NS bifurcation. Although the variations of the multipliers for SB and SN bifurcations are the same, their effects on periodic orbit are different. For example, two periodic orbits can be annihilated by each other for SN bifurcation, while for SB bifurcation, the stability of the symmetric periodic solution will be affected so that the responses of the system are not 
symmetric thereafter. For PD bifurcation, the period of the solution could be doubled at where synchronization is achieved, and the periodic motion of the system will become quasi-periodic after NS bifurcation.

\section{Bifurcation and synchronization analysis}

In this section, bifurcation and synchronization analysis of the impulsively coupled oscillators will be carried out to show the influence of frequency and amplitude of external excitation on the dynamics of the system. For simplicity, we use the abbreviation $n-p: n-q(*)$ in the bifurcation diagram to describe different types of periodic motions of the system, where $n$ is the number of periods of the external excitation in the motion period $T=2 \pi / \omega, p$ is the number of impacts for the first impact oscillator, $q$ is the number of impacts for the second impact oscillator, and "*" is "S" or "AS" representing symmetric or asymmetric periodic motions, respectively.

\subsection{Two-parameter bifurcation and synchronization analysis}

Inspired by [25], we choose the parameter regions $\omega \in[0.6,1.6]$ and $a \in[0.3,1.6]$ to show some typical dynamical behaviors and transitions of the system. Within the selected region, the dynamics of the system is rich so that the APS via various bifurcations could be studied. The diagram of two-parameter bifurcation and synchronization is shown in Fig. 2 which has been divided into ten regimes including a number of SB, PD, and NS bifurcation curves which were obtained by following the corresponding bifurcation points $[33,34]$. As can be seen from the figure, the system exhibits complex dynamics including chaotic motions and periodic windows in the regime marked by (1) at where the system is not anti-phase synchronized. SB bifurcation is observed on the boundary of the regimes (1) and (2), and the response of the system evolves into a period-1 motion with two impacts per period of external excitation. Then the period-1 solution with two impacts in the regime (2) losses stability and bifurcates into a period-2 solution with four impacts in the regime (3) via a PD bifurcation. As the frequency $\omega$ increases, the system regains its stability via a reverse PD bifurcation in the regime (4) at where the period-1 solution with two impacts is observed again. It is worth noting that the anti-phase synchronization of the system is achieved in the regimes (2), (3), and (4), and all of the periodic motions are symmetric. It is seen from the figure that the transition from the regime (4) to (5) is through a SB bifurcation, and the symmetry period-1 solution with two impacts bifurcates into an asymmetry period-1 solution 
with two impacts which is not in APS. As the frequency $\omega$ increases in the regime (5), a SB bifurcation causes the asymmetry period- 1 solution with two impacts losing stability followed by a symmetry period-1 solution with two impacts in the regime (6). Thereafter the system evolves into an asymmetry chaotic motion via a SB bifurcation in the regime (7) which contains chaos, quasi-periodic motion, and periodic windows. The transition between the regimes (7) and (8) is manifested through a NS bifurcation from an asymmetry period-2 solution with two impacts to a quasi-periodic motion. Later on, the regime (8) is followed by a small window of asymmetry period-1 solutions with one impact through a PD bifurcation in the regime (9). Finally, the symmetric period-1 solution with one impact becomes asymmetric after a SB bifurcation as the frequency of external excitation increases. It can be seen from the two-parameter bifurcation diagram that the regimes (2), (3), (4), (6), and (10) which are marked by gray are in APS, and all of them emerge and cease to exist through SB bifurcation. It is therefore that SB bifurcation is crucial for symmetry and APS of the impulsive coupled oscillators. A summary of these transitions are given in Table 1 . The representative phase portraits of the impulsively coupled oscillators for the parameters in these regimes will be given in the next subsection by fixing the amplitude of excitation $a=1$.

Table 1. Summary of the transitions observed from Figure 2.

\begin{tabular}{cccccc}
\hline No. & Regimes & Dynamical transition & APS & APS transition & Bifurcation \\
\hline 1 & $(2) \rightarrow(3)$ & $1-2: 1-2(\mathrm{~S}) \rightarrow 2-4: 2-4(\mathrm{~S})$ & $\mathrm{Y} \rightarrow \mathrm{Y}^{a}$ & $\mathrm{~N}$ & $\mathrm{PD}$ \\
2 & $(3) \rightarrow(4)$ & $2-4: 2-4(\mathrm{~S}) \rightarrow 1-2: 1-2(\mathrm{~S})$ & $\mathrm{Y} \rightarrow \mathrm{Y}$ & $\mathrm{N}$ & $\mathrm{PD}$ \\
3 & $(4) \rightarrow(5)$ & $1-2: 1-2(\mathrm{~S}) \rightarrow 1-2: 1-2(\mathrm{AS})$ & $\mathrm{Y} \rightarrow \mathrm{N}$ & $\mathrm{Y}$ & $\mathrm{SB}$ \\
4 & $(5) \rightarrow(6)$ & $1-2: 1-2(\mathrm{AS}) \rightarrow 1-2: 1-2(\mathrm{~S})$ & $\mathrm{N} \rightarrow \mathrm{Y}$ & $\mathrm{Y}$ & $\mathrm{SB}$ \\
5 & $8 \rightarrow(9)$ & $2-2: 2-2(\mathrm{AS}) \rightarrow 1-1: 1-1(\mathrm{AS})$ & $\mathrm{N} \rightarrow \mathrm{N}$ & $\mathrm{N}$ & $\mathrm{PD}$ \\
6 & $(9) \rightarrow(10)$ & $1-1: 1-1(\mathrm{AS}) \rightarrow 1-1: 1-1(\mathrm{~S})$ & $\mathrm{N} \rightarrow \mathrm{Y}$ & $\mathrm{Y}$ & $\mathrm{SB}$ \\
\hline
\end{tabular}

a"Y" and "N" represent yes and no, respectively.

\subsection{One-parameter bifurcation and synchronization analysis}

In order to investigate the dynamics of the system in detail, one-parameter bifurcation analysis is carried out in this section by varying the frequency of external excitation $\omega$. Bifurcation diagrams were constructed for the frequency range $\omega \in[0.6,1.6]$ by plotting the solution once it converged onto a periodic response, otherwise plotting the next 200 values of displacements if no convergence was found after calculating 2000 periods of external excitation. Fig. 3 presents the 


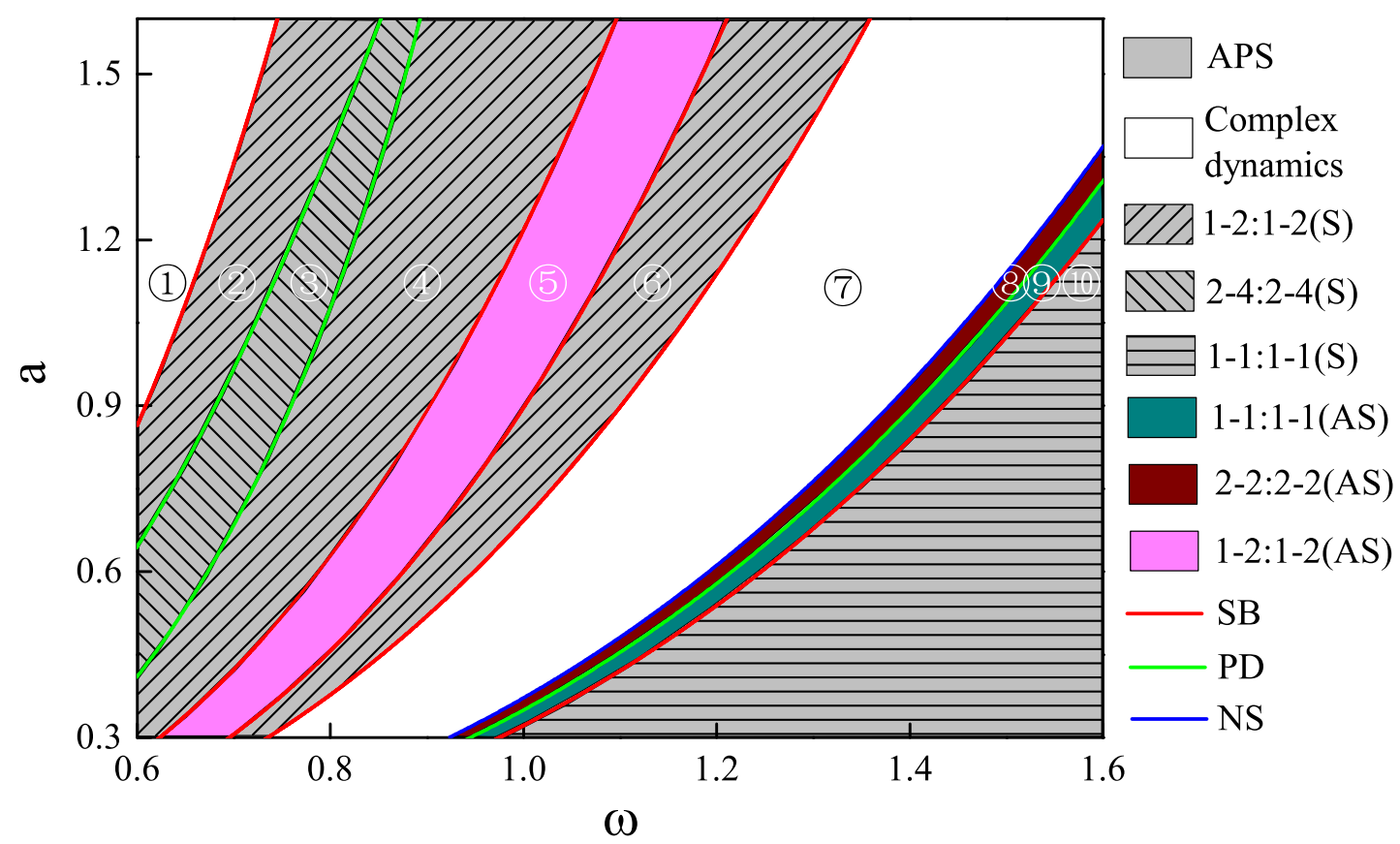

Fig. 2. (Colour online) Two-parameter bifurcation and synchronization diagram obtained for $\xi=0.05, r=0.9$ and $e=1$. The regimes are marked by the numbers (1)-(10), and the regimes in APS are shown by gray shadow. The SB, PD, and NS bifurcations are indicated by red, green, and blue curves, respectively. 
bifurcation diagrams constructed for (a) $x_{1}+x_{2}$, (b) $x_{1}, x_{2}$, and (c) the Lyapunov exponents. The Lyapunov exponents which measure the exponential rates of divergence/convergence of adjacent orbits of the attractors in the state space were calculated by using the Müller's method [35]. The spectrum of the Lyapunov exponent is an effective diagnostic method for monitoring chaotic attractors. The chaotic attractor has at least one positive exponent, and the periodic attractor has only negative exponents with exception of one zero exponent indicating the convergence to another periodic obit. For the quasi-periodic attractor, it has a number of zero exponents and the rest are negative.

As can be seen from Fig. 3, a chaotic regime with a small window of periodic motion is recorded for $\omega \in[0.6,0.671]$ and the system is not in APS. A SB bifurcation is observed for $\omega=0.671$ at where the chaotic solution becomes a symmetric period- 1 solution with two impacts. The time histories and the phase trajectories of the chaotic solution for $\omega=0.67$ are shown in Fig. 4 showing that the APS is not achieved before SB bifurcation. The APS is achieved for $\omega \in(0.671,0.935]$ including a small window of symmetric period-1 motion with two impacts followed by a symmetric period-2 motion with four impacts for $\omega \in(0.708,0.782]$ via a $\mathrm{PD}$ bifurcation, and the system bifurcates again into the symmetric period- 1 motion with two impacts for $\omega \in(0.782,0.935]$ through a reverse $\mathrm{PD}$. The stable symmetric period-1 solution with two impacts loses stability via a SB bifurcation occurring at $\omega=0.935$ followed by a stable asymmetric period-1 solution with two impacts per period of external excitation which is not in APS. The time histories and the phase trajectories of the symmetric period-1 motion with two impact, the symmetric period- 2 motion with four impacts, and the asymmetric period- 1 motion with two impacts for $\omega=0.68, \omega=0.71$, and $\omega=0.94$ are presented in Fig. 5, where the phase plane $\left(x_{1}, x_{2}\right)$ shows APS clearly.

It can be seen from Fig. 3 that the asymmetric period-1 motion with two impacts ceases to exist at $\omega=1.036$, and a symmetric period-1 solution with two impacts emerges afterwards through a SB bifurcation. Hence the system transits from a non-APS regime into a APS one which exists until $\omega=1.144$ followed by a chaotic regime for $\omega \in(1.144,1.43)$ via a SB bifurcation. For $\omega \in[1.487,1.6]$, a stable period-1 solution with one impact in APS is recorded. As the frequency $\omega$ decreases, this periodic solution undergoes a SB bifurcation at $\omega=1.487$ and becomes an asymmetric period- 1 solution with one impact. Then the asymmetric periodic solution bifurcates into an asymmetric period-2 solution with two impacts via a reverse PD at $\omega=1.456$. Time histories and phase trajectories of the asymmetric period- 2 solution with two 


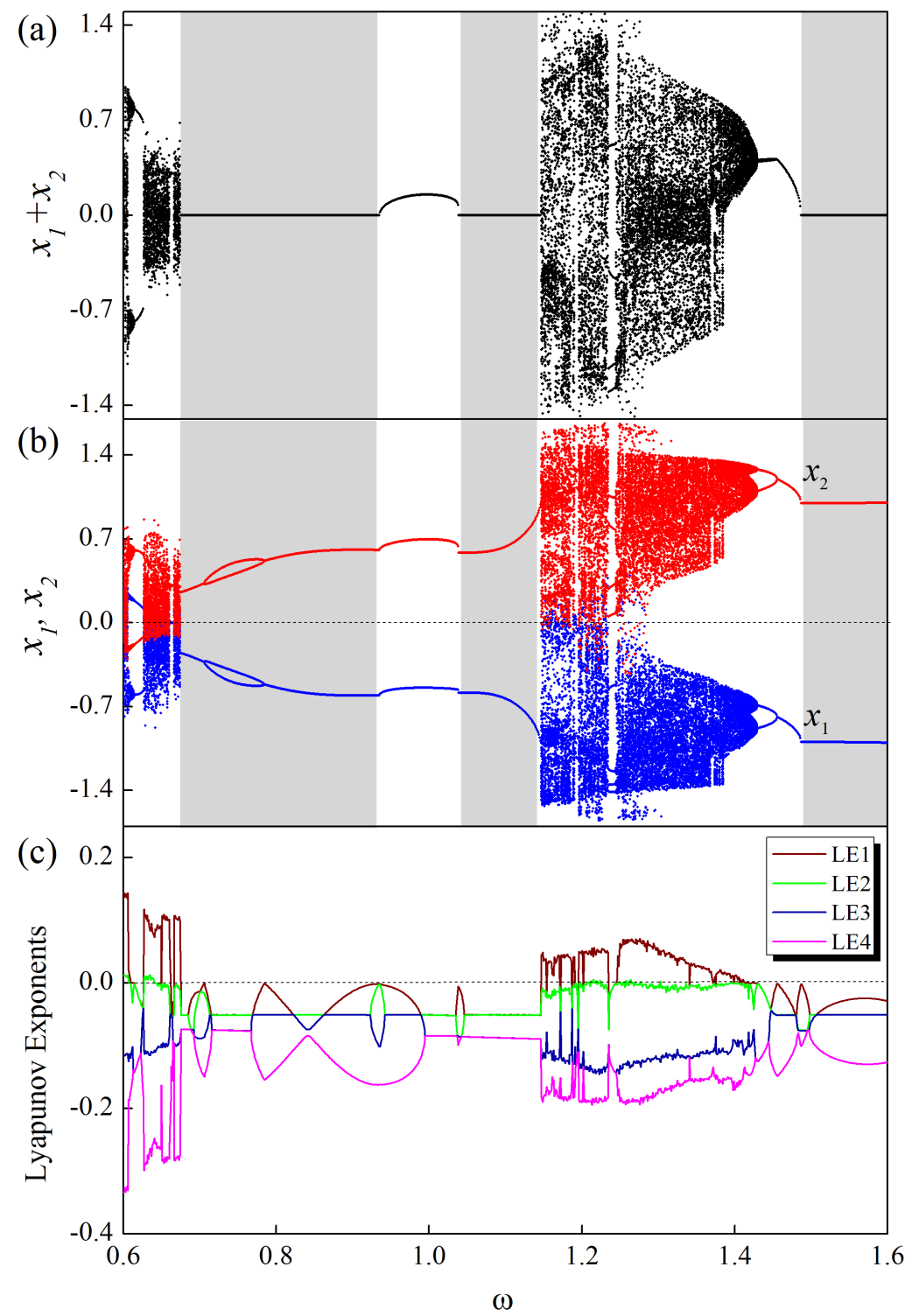

Fig. 3. (Colour online) Bifurcation diagrams obtained for (a) $x_{1}+x_{2}$, (b) $x_{1}$ (blue dots) and $x_{2}$ (red dots), and (c) the Lyapunov exponents by varying the frequency of excitation, $\omega$ calculated for $\xi=0.05, r=0.9, a=1.0$ and $e=1$. The regimes of APS are highlighted by grey shadow, and the Lyapunov exponents are marked in different colours. 
(a)



(b)

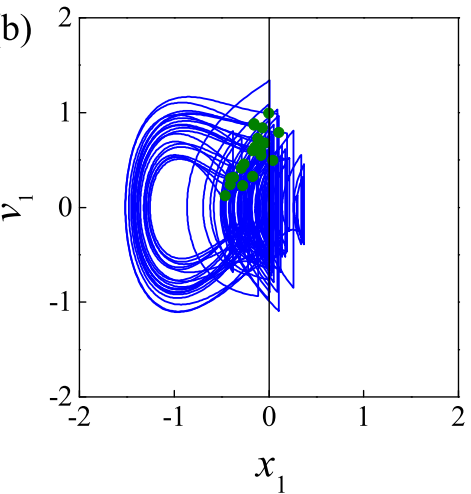

(c)



(d)

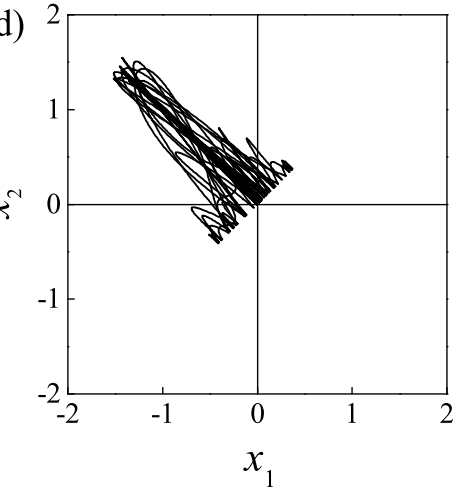

Fig. 4. (Colour online) (a) Time histories of $x_{1}$ (blue line) and $x_{2}$ (red line), (b) the trajectories of mass 1 on the phase plane $\left(x_{1}, v_{1}\right)$, (c) the trajectories of mass 2 on the phase plane $\left(x_{2}, v_{2}\right)$, and $(\mathrm{d})$ the trajectories of the impulsively coupled oscillators on the phase plane $\left(x_{1}, x_{2}\right)$ for the chaotic solution calculated for $\xi=0.05, r=0.9, a=1.0, e=1$, and $\omega=0.67$. Poincaré sections are marked by green dots. 

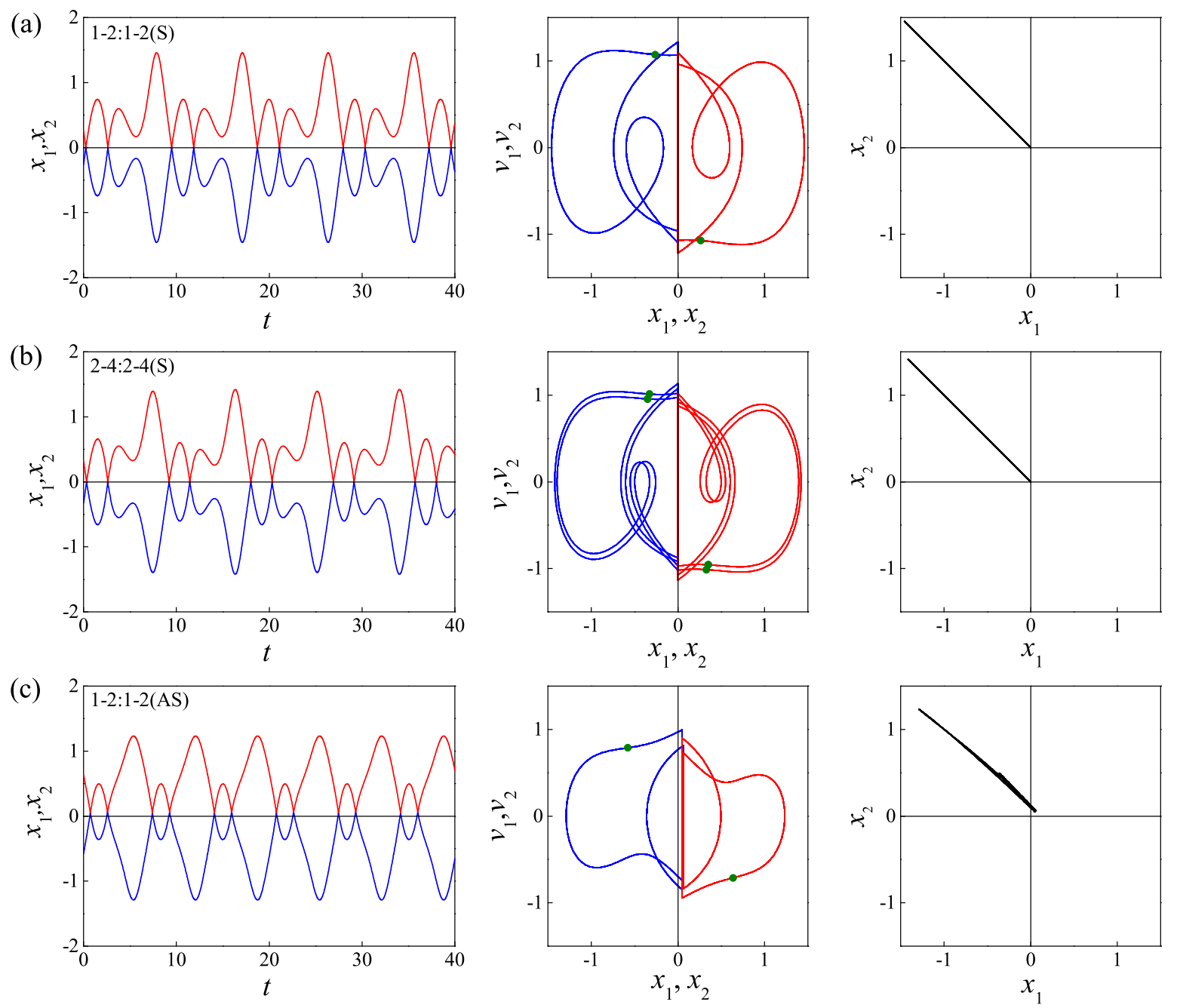

Fig. 5. (Colour online) Time histories of $x_{1}$ (blue line) and $x_{2}$ (red line), the trajectories of mass 1 on the phase plane $\left(x_{1}, v_{1}\right)$ (blue line) and the trajectories of mass 2 on the phase plane $\left(x_{2}, v_{2}\right)$ (red line), and the trajectories of the impulsively coupled oscillators on the phase plane $\left(x_{1}, x_{2}\right)$ for (a) the symmetric period-1 solution with two impacts $(\omega=0.68)$, (b) the symmetric period-2 solution with four impacts $(\omega=0.71)$, and (c) the asymmetric period-1 solution with two impacts $(\omega=0.94)$ calculated for $\xi=0.05, r=0.9, a=1.0, e=1$. Poincaré sections are marked by green dots. 

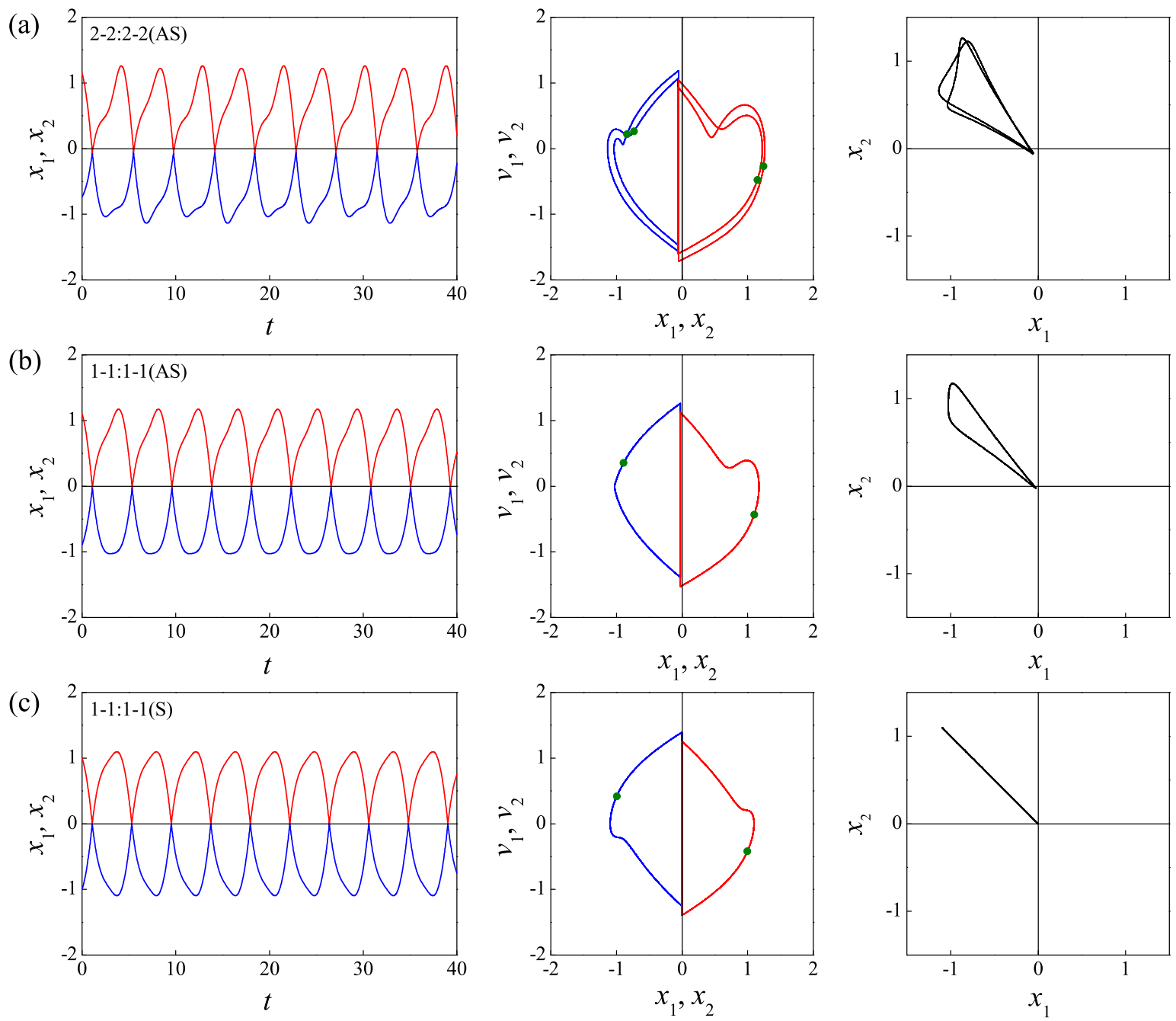

Fig. 6. (Colour online) Time histories of $x_{1}$ (blue line) and $x_{2}$ (red line), the trajectories of mass 1 on the phase plane $\left(x_{1}, v_{1}\right)$ (blue line) and the trajectories of mass 2 on the phase plane $\left(x_{2}, v_{2}\right)$ (red line), and the trajectories of the impulsively coupled oscillators on the phase plane $\left(x_{1}, x_{2}\right)$ for (a) the asymmetric period-2 solution with two impacts ( $\omega=1.45$ ), (b) the asymmetric period- 1 with one impact $(\omega=1.48)$, and (c) the symmetric period- 1 with one impact $(\omega=1.49)$ calculated for $\xi=0.05, r=0.9, a=1, e=1$. Poincaré sections are marked by green dots. 
impacts at $\omega=1.45$, the asymmetric period- 1 solution with one impact at $\omega=1.48$, and the symmetric period-1 solution with one impact at $\omega=1.49$ are shown in Fig. 6. The asymmetric period-2 solution is recorded for $\omega \in[1.43,1.456)$ and bifurcates into a quasi-periodic solution at $\omega=1.43$ via a NS bifurcation. As the frequency $\omega$ decreases, the quasi-periodic solution exists shortly following by the chaotic solutions for $\omega \in(1.144,1.43)$. Fig. 7 presents the time histories and the phase trajectories of the quasi-periodic solution at $\omega=1.43$ at where the zoom-in of the Poincaré sections are shown in the blow-up windows.

(a)


Fig. 7. (Colour online) (a) Time histories of $x_{1}$ (blue line) and $x_{2}$ (red line), (b) the trajectories of mass 1 on the phase plane $\left(x_{1}, v_{1}\right)$, (c) the trajectories of mass 2 on the phase plane $\left(x_{2}, v_{2}\right)$, and (d) the trajectories of the impulsively coupled oscillators on the phase plane $\left(x_{1}, x_{2}\right)$ for the quasi-periodic solution calculated for $\xi=0.05, r=0.9, a=1.0, e=1$, and $\omega=1.43$. Poincaré sections marked by green dots are shown in blow-up windows. 


\section{Conclusions}

This paper studies the dynamics and the synchronization in two mechanical oscillators coupled by impacts which can be considered as a class of state-dependent impulsively coupled oscillators. The two identical oscillators are harmonically excited in a counter phase, and the synchronous (APS) and the asynchronous motions of the system were considered. Event-based hybrid system modeling and stability analysis were given and the numerical continuation method was employed to carry out bifurcation analysis. One- and two-parameter bifurcations of the system were studied by varying the amplitude and the frequency of external excitation. From the two-parameter bifurcation study, we would be able to identify the regimes of APS in a two-parameter domain $(\omega, a)$. Furthermore, the conclusion that SB bifurcation dominates the transition from APS to asynchronous motion was verified by following bifurcation curves using the continuation technique.

Two-parameter bifurcation was carried out by varying the frequency and the amplitude of external excitation, and ten regimes and a number of SB, PD, and NS bifurcation curves were obtained. It has shown that all the regimes in APS emerge and cease to exist through SB bifurcation. The transition between the regime of complex dynamics and the regime of asymmetric period-2 solution with two impacts is manifested through NS bifurcation. We also observed that the system underwent quasi-periodic motions before completely changed to chaos as the frequency of excitation decreased.

One-parameter bifurcation was studied for the frequency range $\omega \in[0.6,1.6]$ with a fixed amplitude $a=1$. The Lyapunov exponents were presented with the bifurcation diagrams in order to interpret different bifurcation scenarios. From one-parameter bifurcation study, we would be able to study the dynamical transitions of the system by observing its time history and phase portrait. Numerical simulations have shown that the system could exhibit complex phenomena, including symmetry and asymmetry periodic solutions, quasi-periodic solutions and chaotic solutions. In addition, we could conclude that the symmetric periodic solutions which are in APS may emerge after SB bifurcation.

\section{Acknowledgements}

This work is partially supported by the National Natural Science Foundation of China (Grant nos. 11402224, 11202180, 61273106 and 11171290), the Natural Science Foundation of 
Jiangsu Province of China (Grant no. BK20151295), the Qin Lan Project of the Jiangsu Higher Education Institutions of China, and the Jiangsu Overseas Research and Training Program for University Prominent Young and Middle-aged Teachers and Presidents. We also thank Mr. Marcin Kapitaniak for helpful discussion.

\section{References}

[1] Pecora LM, Carroll TL. Synchronization in chaotic systems. Phys Rev Lett 1990;64:821-4.

[2] Pikovsky A, Rosenblum M, Kurths J. Synchronization: a universal concept in nonlinear science. Cambridge: Cambridge University Press; 2001.

[3] Strogatz SH. From Kuramoto to Crawford: exploring the onset of synchronization in populations of coupled oscillators. Physica D 2000;143:1-20.

[4] Zhou J, Cheng XH, Xiang L, Zhang YC. Impulsive control and synchronization of chaotic systems consisting of Van der Pol oscillators coupled to linear oscillators. Chaos Solitons Fractals 2007;33: 607-16.

[5] Rosenblum MG, Pikovsky AS, Kurths J. Phase synchronization of chaotic oscillators. Phys Rev Lett 1996;76: 1804-7.

[6] Zhang LP, Jiang HB, Bi QS. Reliable impulsive lag synchronization for a class of nonlinear discrete chaotic systems. Nonlinear Dyn 2010;59:529-34.

[7] Wang QY, Lu QS. Adaptive lag synchronization in coupled chaotic systems with unidirectional delay. Int J Non-Linear Mech 2010;45:640-6.

[8] Zhang LP, Jiang HB. Impulsive generalized synchronization for a class of nonlinear discrete chaotic systems. Commun Nonlinear Sci Numer Simulat 2011;16:2027-32.

[9] Zhang YP, Sun JT. Chaotic synchronization and anti-synchronization based on suitable separation. Phys Lett A 2004;330:442-7.

[10] Li RH, Xu W, Li S. Anti-synchronization on autonomous and non-autonomous chaotic systems via adaptive feedback control. Chaos Solitons Fractals 2009;40:1288-96. 
[11] Hugenii C. Horoloqium Oscilatorium, Apud F. Muquet, Parisiis, 1673, English translation: The Pendulum Clock, Iowa State University Press, Ames, 1986.

[12] Czolczynski K, Perlikowski P, Stefanski A, Kapitaniak T. Clustering and synchronization of n Huygens' clocks. Physica A 2009;388:5013-23.

[13] Liu WQ. Antiphase synchronization in coupled chaotic oscillators. Phys Rev E 2006;73:057203.

[14] Rene Y. Synchronization dynamics in a ring of four mutually inertia coupled self-sustained electrical systems. Physica A 2006;366:187-96.

[15] Barrón MA, Sen M. Synchronization of four coupled van der Pol oscillators. Nonlinear Dyn 2009;56:357-67.

[16] Perlikowski P, Yanchuk S, Wolfrum M, Stefanski A, Mosiolek P, Kapitaniak T. Routes to complex dynamics in a ring of unidirectionally coupled systems. Chaos 2010;20:013111.

[17] Kapitaniak M, Perlikowski P, Kapitaniak T. Synchronous motion of two vertically excited planar elastic pendula. Commun Nonlinear Sci Numer Simulat 2013;18:2088-96.

[18] Han XP, Lu JA, Wu XQ. Synchronization of impulsively coupled systems. Int J Bifur Chaos 2008;18:1539-49.

[19] Yang M, Wang YW, Xiao JW, Wang HO. Robust synchronization of impulsively-coupled complex switched networks with parametric uncertainties and time-varying delays. Nonlinear Anal Real World Appl 2010;11:3008-20.

[20] Jiang HB, Bi QS. Contraction theory based synchronization analysis of impulsively coupled oscillators. Nonlinear Dyn 2012;67:781-91.

[21] Jiang HB, Zhang LP, Yu JJ. Complex dynamics analysis of impulsively coupled Duffing oscillators with ring structure. Chin Phys B 2015;24:020502.

[22] Jiang HB, Yu JJ, Zhou CG. Consensus of multi-agent linear dynamic systems via impulsive control protocols. Int J Systems Sci 2011;42:967-76.

[23] Jiang HB, Bi QS. Impulsive synchronization of networked nonlinear dynamical systems. Phys Lett A 2010;374:2723-9. 
[24] Jiang HB, Bi QS, Zheng S. Impulsive consensus in directed networks of identical nonlinear oscillators with switching topologies. Commun Nonlinear Sci Numer Simul 2012;17:378-87.

[25] Blazejczyk-Okolewska B, Brindley J, Czolcznski K, Kapitaniak T. Antiphase synchronization of chaos by noncontinuous coupling two impact oscillators. Chaos Solitons Fractals $2001 ; 12: 1823-6$.

[26] Luo ACJ. On the symmetry of solutions in non-smooth dynamical systems with two constraints. J Sound Vib, 2004;273:1118-26.

[27] Yue Y, Xie JH. Symmetry and bifurcations of a two-degree-of-freedom vibro-impact system. J Sound Vib, 2008;314:228-45.

[28] Luo GW, Lv XH, Shi YQ. Vibro-impact dynamics of a two-degree-of freedom periodicallyforced system with a clearance: diversity and parametermatching of periodic-impact motions. Int J Non-Linear Mech, 2014;65:173-95.

[29] Li Q, Wei L, Tan J, Xi J. Double grazing periodic motions and bifurcations in a vibroimpact system with bilateral stops. Abstr Appl Anal, 2014;2014:642589.

[30] Thota P, Dankowicz H. TC-HAT: A novel toolbox for the continuation of periodic trajectories in hybrid dynamical systems. SIAM J Appl Dyn Syst 2008;7:1283-322.

[31] Páez Chávez J, Pavlovskaia E, Wiercigroch M. Bifurcation analysis of a piecewise-linear impact oscillator with drift. Nonlinear Dyn 2014;77:213-27.

[32] di Bernardo M, Budd CJ, Champneys AR, Kowalczyk P. Piecewise-smooth Dynamical Systems: Theory and Applications. New York: Springer-Verlag; 2008.

[33] Dankowicz H, Schilder F. An extended continuation problem for bifurcation analysis in the presence of constraints. J Comput Nonlinear Dynam 2011;6:031003.

[34] Dankowicz H, Schilder F. Recipes for continuation. Philadelphia: Society for Industrial and Applied Mathematics; 2013.

[35] Müller PC. Calculation of Lyapunov exponents for dynamics systems with discontinuities. Chaos Solitons Fractals 1995;5:1671-81. 